\title{
DERIVATION OF THE FORMULA FOR THE FILTRATION COEFFICIENT BY APPLICATION OF POISEUILLE'S LAW IN MEMBRANE TRANSPORT
}

\author{
MARIA JARZYŃSKA ${ }^{1}$, MARIUSZ PIETRUSZKA ${ }^{2}$ \\ ${ }^{1}$ Technical High School of Environment Developing \\ Broniewskiego 16, 97-300 Piotrków Trybunalski, Poland \\ e-mail: jarzynska@op.pl \\ ${ }^{2}$ Faculty of Biology and Environmental Protection, University of Silesia \\ Jagiellońska 28, 40-032 Katowice, Poland
}

(Received: November 21, 2008. Accepted: February 18, 2009)

\begin{abstract}
On the basis of Kedem-Katchalsky equations a mathematical analysis of volume flow $\left(J_{v}\right)$ of a binary solution through a membrane $(M)$ is presented. Two cases of transport generators have been considered: hydrostatic $(\Delta p)$ as well as osmotic $(\Delta \Pi)$ pressure difference. Based on the Poiseuille's law we derive the formula for the membrane filtration coefficient $\left(L_{p}\right)$ which takes into account the membrane properties, kinetic viscosity and density of a solution flowing across the membrane. With use of this formula we have made model calculations of the filtration coefficient $L_{p}$ and volume flow $J_{v}$ for a polymer membrane in the case when the solutions on both sides of the membrane are mixed.
\end{abstract}

KEY WORDS: membrane, filtration coefficient, viscosity coefficient, Poiseuille's law.

\section{INTRODUCTION}

Synthetic polymer membranes have been widely applied in membrane transport research, engineering and also in

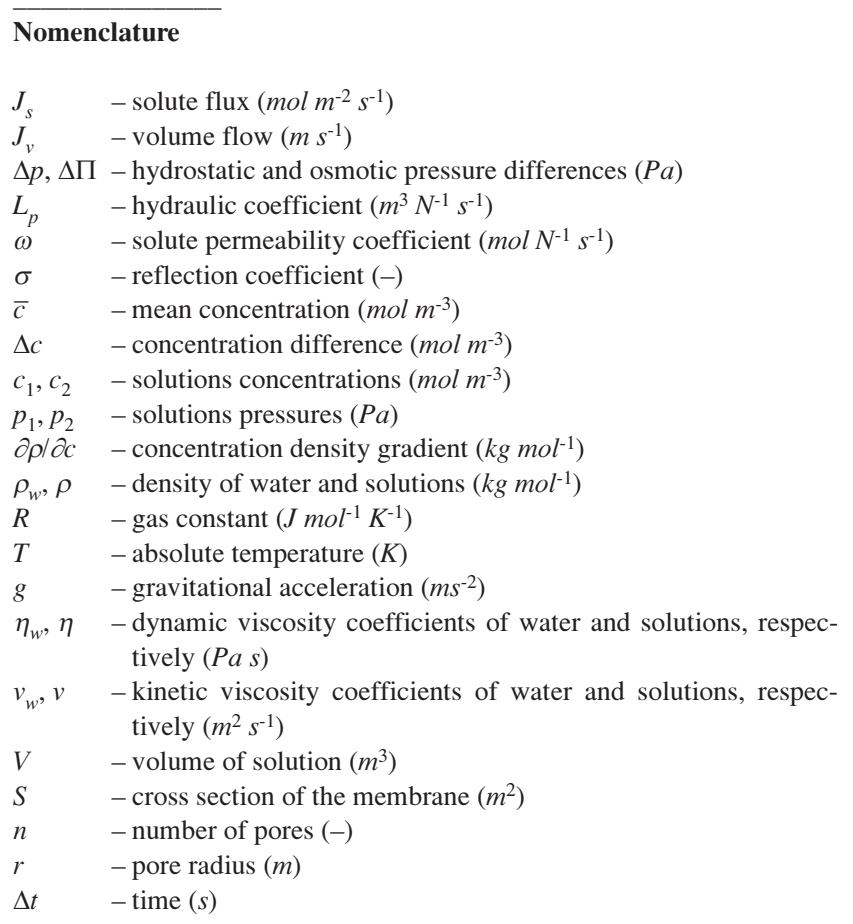

medicine, in separation techniques field. Transport through such membranes is well described by the Kedem-Katchalsky $(\mathrm{K}-\mathrm{K})$ equations for volume flow $\left(J_{v}\right)$ and solute flow $\left(J_{s}\right)$. These equations have been derived from the principles of linear non-equilibrium thermodynamics (LNET) and can be well applied to both synthetic and biological membranes. The classical version of the K-K equations applies to homogeneous membrane systems with well mixed solutions. For the case of poorly stirred solutions the lack of mixing leads to a formation of concentration boundary layers (CBLs) in the vicinity of the membrane. Such layers participate in the transport and therefore the K-K equations have to be modified.

The aim of this paper is to investigate the membrane transport described by the K-K equations for the case in which the transport is generated by the hydraulic pressure gradient $(\Delta p)$ as well as the osmotic pressure gradient $(\Delta \Pi)$. We consider the special case of ideal mixing of solutions. On the basis of the Darcy's law and the Poiseuille's law we derive the formula for the hydraulic coefficient $L_{p}$ (also called filtration coefficient), as a function of dynamic viscosity, for fixed concentration of the solution flowing across the membrane. To test the validity of the formula and to get more insight into the membrane transport we consider a model of a cell, which consists of a single membrane that separates two binary solutions, having concentrations $c_{1}$ and $c_{2}$ and pressures $p_{1}$ and $p_{2}$ respectively. We assume that the following conditions are fulfilled for 
the cell: $c_{1}>c_{2}, p_{1}>p_{2}$ and $T=$ const. From the Van't Hoff equation the osmotic pressure difference reads $\Delta \Pi=R T \Delta c$ (Kedem and Katchalsky 1958; Katchalsky and Curran 1965; Kedem and Katchalsky 1963; Gumiński 1962; Dołowy et al. 2003) and the hydrostatic pressure difference $\Delta p$ $=p_{1}-p_{2}$. For this model we also derive the formula for volume flow $J_{v}$ and include the influence of the concentration of the solution on $L_{p}$ and $J_{v}$. We discuss the results for two cases of transport: generated either by $\Delta p$ or $\Delta \Pi$. The results presented below may apply to polymer membranes, used in medicine, and aqueous glucose solutions for the case when the transport is stationary and the solutions on both sides of the membrane are well mixed.

\section{The Kedem-Katchalsky equations}

Membrane transport for binary non-electrolyte solutions, generated by the hydrostatic pressure difference $(\Delta p)$ and the osmotic pressure difference $(\Delta \Pi)$, can be described by the Kedem-Katchalsky equations. The K-K equations have been derived from the principles of linear thermodynamics of irreversible processes. Such transport is described by the equations for the volume flow, $\left(J_{v}\right)$ and the solute flow $\left(J_{s}\right)$ (Kedem O., Katchalsky A. 1958; Katchalsky A., Curran P.F. 1965; Kedem O., Katchalsky A. 1963):

$$
\begin{aligned}
& J_{v}=L_{p} \Delta p-L_{p} \sigma \Delta \Pi \\
& J_{s}=\omega \Delta \Pi+(1-\sigma) \bar{c} J_{v}
\end{aligned}
$$

where $\bar{c}$ stands for mean concentration, $\bar{c} \approx \frac{1}{2}\left(c_{1}+c_{2}\right)$

and $\left(L_{p}, \sigma, \omega\right)$ are coefficients of filtration, reflection and permeation, respectively. The above equations have widely been used in research on substance permeability through artificial and biological membranes (Ginzburg and Katchalsky 1963). The application of the K-K equations in their classical version is limited to membrane systems with two-component solutions, sufficiently diluted and well stirred (Koter 2005; Bacchin et al. 2006; Ślęzak et al. 2004; Jarzyńska 2005).

\section{The membrane system}

The system under consideration consists of a 1-membrane cell, presented in the sketch in Figure 1. The membrane

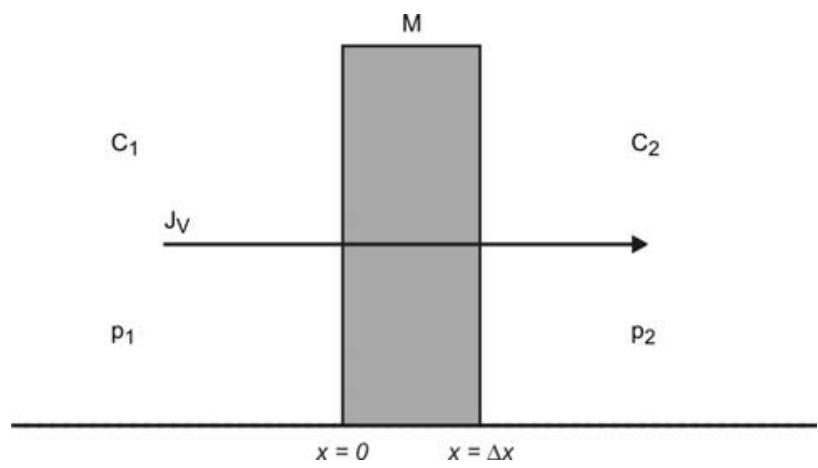

Fig. 1. Sketch of a membrane cell: $M$ is the homogeneous membrane of thickness $\Delta x ; c_{1}$ and $c_{2}$ are the concentrations of solutions in compartments separated by the membrane, $\Delta c=c_{1}-c_{2} ; p_{1}$ and $p_{2}$ are the values of the hydraulic pressure in appropriate compartments, $\Delta p=p_{1}-p_{2} ; J_{v}$ is the solution volume flux through the membrane $M$.
$(M)$ divides the cell into two compartments filled with binary non-electrolyte solutions of concentration $c_{1}$ and $c_{2}$ and pressures $p_{1}$ and $p_{2}$ in isothermal conditions. The osmotic pressure difference between the solutions $(\Delta \Pi)$ is given by Van't Hoff equation $\Delta \Pi=R T\left(c_{1}-c_{2}\right)$, and the mechanical pressure difference is $\Delta p=p_{1}-p_{2}$. The membrane is characterized by following parameters: filtration coefficient $\left(L_{p}\right)$, reflection coefficient $(\sigma)$ and permeability coefficient $(\omega)$.

Transport across the membrane depends on the rate of mixing of the solutions. When the solutions are poorly or not at all mixed, the effect of forming of boundary layers $h$ and $l$ in the vicinity of the membrane occurs and both the membrane and the layers $(h / M / l)$ participate in transport. In the case when the solutions are mixed, only the membrane takes part in transport. In this paper we restrict ourselves to the second case assuming equally mixed solutions. We also assume that the transport is stationary.

\section{Derivation of the filtration coefficient $\left(L_{p}\right)$ formula}

The filtration coefficient describes the ability of a pore environment to permeate fluid when a pressure gradient exists, while filtration is the fluid ability to permeate through the pore environment.

In 1856 Henry Darcy, on the basis of his experiments, derived the linear filtration law, called nowadays Darcy's law. He showed that for laminar flow the volume of fluid $(V)$, permeating through a pore medium, is proportional to the cross-section area $(S)$ of the medium, flow time $(t)$, the hydraulic pressure drop $(\Delta p)$ and the filtration coefficient $\left(L_{p}\right)$, called also the hydraulic conductivity of a membrane. According to the Darcy's law the filtration coefficient describes not only the pore medium itself, but also the medium-fluid pair e.g. membrane-solution, membrane-water and so on.

Let us consider the Kedem-Katchalsky equation (1) for the volume flow $J_{v}$ through a membrane. When the flow is forced only by hydrostatic pressure difference $\Delta p(\Delta \Pi=$ $0),(1)$ reads

$$
J_{v}=L_{p} \Delta p
$$

One can rewrite Eq. (3) in the form

$$
J_{v}=\frac{\Delta V}{S \Delta t},
$$

where $\Delta V$ is the volume of the solute permeating through the area $S$ of the pore in time $\Delta t$.

On the basis of the Poiseuille's law the volume $\Delta V$ is expressed as follows

$$
\Delta V=\frac{1}{\eta} \frac{\Delta p r^{4} \pi \Delta t}{8 l},
$$

where

$\Delta V$ - volume flow rate,

$r$ - inner radius of the capillary (pore),

$\eta$ - fluid dynamic viscosity coefficient,

$\Delta p$ - pressure difference at the ends of the capillary,

$l$ - length of the capillary.

The Poiseuille's law describes the relation between volume flow rate, viscosity, pressure gradient, that causes the fluid flow, and the capillary parameters (its length and ra- 
dius). For the stationary, laminar flow of viscous fluid through a cylindrical pipe (i.e. a pore with constant, circular cross-section) the volume flow rate is proportional to the pressure gradient along the pipe and thus to the pressure difference at the ends of the pipe.

Taking into (for $n$ pores) account Eq. (5) equation (4) takes on the form

$$
J_{v}=n \frac{r^{2}}{8 \ln } \Delta p
$$

From (3) and (6) we get

$$
L_{p}=n \frac{r^{2}}{8 \eta \Delta x},
$$

where $\Delta x$ is the thickness of the membrane and $\eta$ is the dynamic viscosity of the fluid. In order to determine the value of $L_{p}$ for an arbitrary solution and a membrane one can use the value of the filtration coefficient obtained for water and the same membrane $\left(L_{p w}\right)$, taking into account the change of viscosity and density while changing concentration of investigated solution. From Eq. (7) the hydraulic conductivity of the membrane and solvent (water) reads

$$
L_{p w}=n \frac{r^{2}}{8 \eta_{w} \Delta x},
$$

where $\eta_{w}$ is the viscosity of water (solvent), $n$ is the number of pores within the membrane.

If we express the fluid kinetic viscosity $(v)$ by fluid dynamic viscosity $(\eta)$ and fluid density $(\rho)$

$$
v=\frac{\eta}{\rho}
$$

the formula for the membrane filtration coefficient $L_{p}$ for the solution with a given concentration, is of the form

$$
L_{p}=L_{p w} \frac{v_{w} \rho_{w}}{v \rho} \text { or } L_{p}=L_{p w} \frac{\eta_{w}}{\eta}
$$

where

$v$ - solution kinetic viscosity,

$\rho$-solution density.

Next, inserting (10) into (1) the K-K equation for the volume flow $J_{v}$ is given by

$$
J_{v}=L_{p w} \frac{v_{w} \rho_{w}}{v \rho}(\Delta p-\sigma \Delta \Pi) .
$$

In order to test Eq. (11) we have performed the calculations for the membrane cell presented in Figure 1. A polymer (cellophane) membrane, that may be used in medicine, separates two aqueous glucose solutions having concentrations $c_{1}$ and $c_{2}$. The transport parameters of such a membrane are (Ślęzak et al. 2004): hydraulic conductivity $L_{p}=$ $5 \times 10^{-12} \mathrm{~m}^{3} \mathrm{~N}^{-1} \mathrm{~s}^{-1}$, reflection coefficient $\sigma=0.068$, permeability coefficient $\omega=8 \times 10^{-10} \mathrm{~mol} \times N^{-1} \mathrm{~s}^{-1}$. The difference of concentrations of the solutions in two compartments, forcing the transport through the membrane, is $\Delta c=0.1$ $m o l m^{-3}, c_{1}=n \Delta c n,=1,2,3, \ldots, 201$; kinetic viscosity and density in solutions for corresponding concentrations fulfill the conditions $v_{(n)}=v_{w}+n \Delta v$ and $\rho_{(n)}=\rho_{(w)}+n \Delta \rho$, where $v_{w}=1.012 \times 10^{-6} \mathrm{~m}^{2} \times \mathrm{s}^{-1}$ and $\rho_{w}=998 \mathrm{~kg} \times \mathrm{m}^{-3}$ are water kinetic viscosity and water density, respectively; the values of the increase of kinetic viscosity and density with increa- sing concentration of the solution are $\Delta v=0.00004 \mathrm{~m}^{2} \times \mathrm{s}^{-1}$ and $\Delta \rho=0.006 \mathrm{~kg} \times \mathrm{m}^{-3}$, respectively. The values of $\Delta v$ and $\Delta \rho$ have been empirically determined (Ślęzak et al. 2004). The remaining parameters are as follows: gas constant $R=8.31 \mathrm{~J} \times \mathrm{mol}^{-1} \mathrm{~K}^{-1}$; gravitational acceleration $g=$ $9.81 \mathrm{~m} \times \mathrm{s}^{-2}$. All calculations have been carried out for isothermal conditions at temperature $T=295 \mathrm{~K}$.

\section{RESULTS AND DISCUSSION}

\section{Dependence $L_{p}=f(c)$}

In Figure 2 we present the results obtained for the filtration coefficient $L_{p}$ as a function of concentration of the solution $c$. The calculations have been carried out with the use of Eq. (10) with the threshold value of water filtration coefficient: $L_{p}=5 \times 10^{-12} \mathrm{~m}^{3} \mathrm{~N}^{-1} \mathrm{~s}^{-1}$.

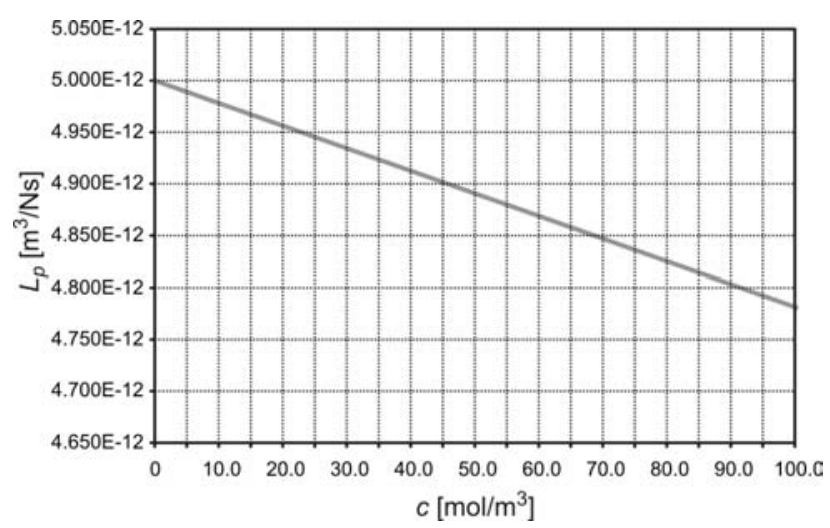

Fig. 2. Graphic illustration of the dependence $L_{p}=f(c)$. The membrane filtration coefficient is inversely proportional to the concentration of the solution.

\section{Dependence $J_{v}=f\left(L_{p}\right)$}

The results for $L_{p}$ as a function of the solution flux $\left(J_{v}\right)_{\Delta p=0}$ flowing through the membrane are presented in Figure 3. Transport is generated by the osmotic pressure gradient $\Delta \Pi(\Delta p=0)$ for the case when the solutions concentration difference changes from $\Delta c=0 \frac{\mathrm{mol}}{\mathrm{m}^{3}}$ to $\Delta c=100 \frac{\mathrm{mol}}{\mathrm{m}^{3}}$.

The calculations of $J_{v}$ have been carried out using Eq. (11).

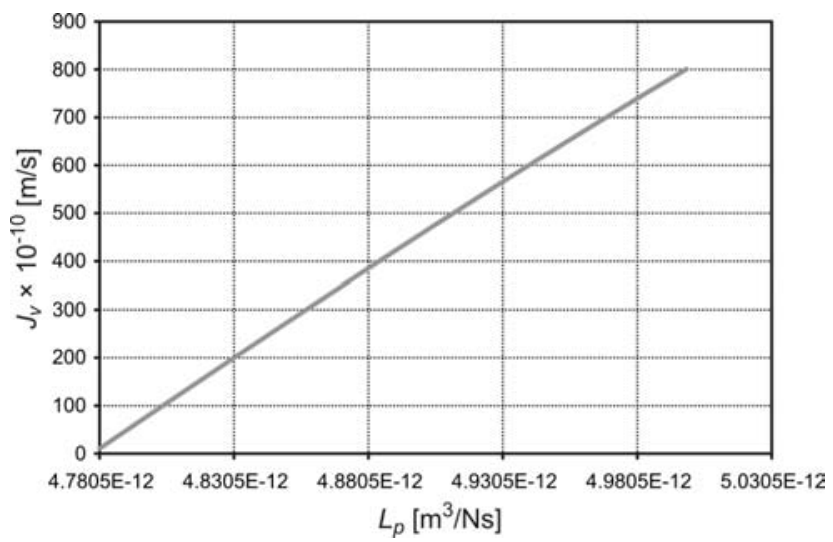

Fig. 3. Graphic illustration of the dependence $\left|J_{v}\right|=f\left(L_{p}\right)$. The gradient of the solutions concentration, causing the flow, changes in the range from $\Delta c=0 \frac{\mathrm{mol}}{\mathrm{m}^{3}}$ to $\Delta c=100 \frac{\mathrm{mol}}{\mathrm{m}^{3}}$. 


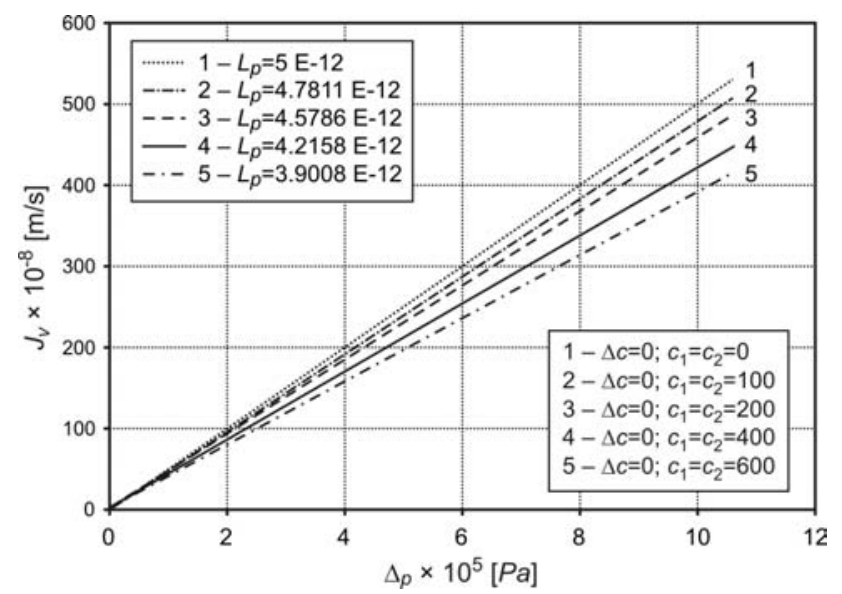

Fig. 4. Graphic illustration of the dependence $J_{v}=f(\Delta p), \Delta \Pi=0$. All plots are obtained for different values of solutions concentration with the assumption $c_{1}=c_{2}\left(\mathrm{~mol} / \mathrm{m}^{3}\right)$. The influence of the solution concentration on the filtration coefficient $L_{p}$ is taken into account.

\section{Dependence $J_{v}=f\left(\Delta_{p}\right)_{\Delta \Pi=0}$}

The dependence of the volume flow $J_{v}$ on the mechanical pressure difference $\Delta p$ is presented in Figure 4. In this case $\Delta \Pi=0$ thus the only source of transport is the hydraulic pressure. The five plots correspond to the different values of the solutions concentrations with $c_{1}=c_{2}$. We see that the volume flow decreases with increasing concentration of the solutions.

\section{Dependence $J_{v}=f\left(\Delta_{c}\right)_{\Delta p=0}$}

In this section we show how the volume flow $J_{v}$ depends on the solution concentration for the case when the flow is generated by the osmotic pressure difference $\Delta \Pi(\Delta p=0)$. In Figure 5, plot 1 shows the results obtained for the case when the value of the filtration coefficient $L_{p}$ does not depend on the concentration of the solution $\left(L_{p}=\right.$ const $)$, plot 2 the results for the case when this dependence is taken into account.

\section{CONCLUSIONS}

In this paper we have presented the results of the model calculations of the Kedem-Katchalsky equations applied to a 1-membrane cell filled with a non-electrolyte binary solution. To be specific, we have used the polymer membrane applied in medicine and the well-mixed aqueous glucose solution. On the basis of the Darcy's and Poiseuille's laws we have derived new the formula for the filtration coefficient $\left(L_{p}\right)$ and then, using the K-K equations, the formula for the volume flow $J_{v}$. It was found out that $L_{p}$ depends essentially on the properties of the membrane and the fluid properties, i.e. it is inversely proportional to kinetic viscosity and density of permeating fluid. The obtained results are in agreement with the Darcy's law.

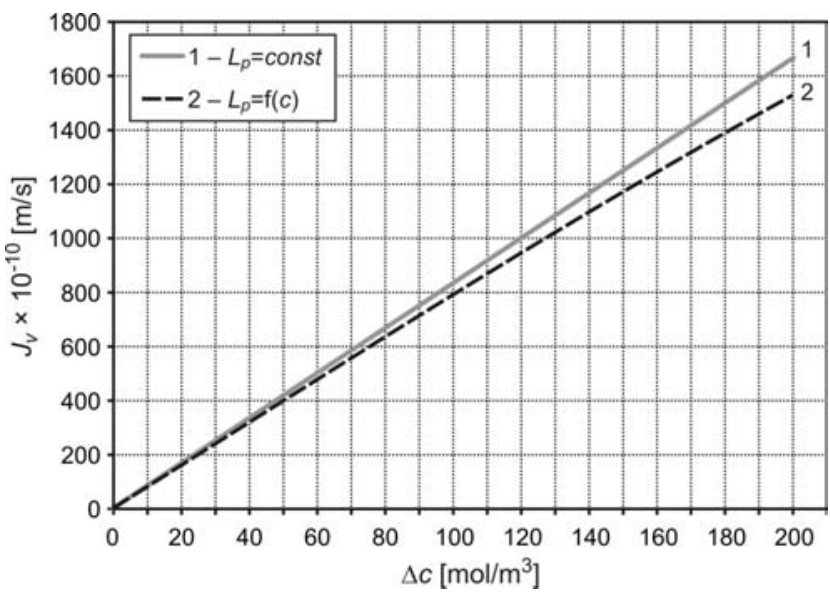

Fig. 5. Graphic illustration of the dependence $J_{v}=f(\Delta c)_{\Delta p=0}, \Delta p=0$. Plot 1 is taken for $L_{p}=$ const, plot 2 with the influence of the solution concentration on $L_{p}: 4.9977 \times 10^{-12} m^{3} N^{-1} s^{-1}, \ldots, 4.5786 \times 10^{-12} m^{3} N^{-1} s^{-1}$.

It tunes out that, the filtration coefficient $L_{p}$ not only depends on physical or chemical properties of the membrane itself but also on the properties of the fluid flowing through the membrane.

\section{LITERATURE CITED}

BACCHIN P., ESPINASSE B., BESSIERE Y., FLETCHER D.F., AIMAR P. 2006. Numerical simulation of colloidal dispersion filtration; description of critical flux and comparison with experimental results, Desalination 192: 74-81.

DOŁOWY K., SZEWCZYK A., PIKUŁA S. 2003. Biological membranes, page 109. Scientific Publisher "Śląsk" Katowice-Warszawa. (in Polish)

GINZBURG B.Z., KATCHALSKY A. 1963. The frictional coefficients of the flows on non-electrolytes through artificial membrane. J. Gen. Physiol. 47: 403-418.

GUMIŃSKI K. 1962. Thermodynamics of Irreversible Processes. PWN, Warsaw. pp 57-63. (in Polish)

JARZYŃSKA M. 2005. Mechanistic equations for membrane substance transport are consistent with Kedem - Katchalsky equations. J. Membr. Sci. 263: 162-163.

KATCHALSKY A., CURRAN P.F. 1965. Non-equilibrium Thermodynamics in Biophysics. Harvard University Press, Cambridge, MA.

KEDEM O., KATCHALSKY A. 1958. Thermodynamics analysis of the permeability of biological membranes to non-electrolytes. Biochim. Biophys. Acta 27: 229-246.

KEDEM O., KATCHALSKY A. 1963. Permeability of composite membranes. Trans. Faraday. Soc. 59 (Part 1-3).

KOTER S. 2005. The Kedem-Katchalsky equations and the sieve mechanism of membrane transport. J. Membr. Sci. 246: 109-111.

ŚLĘZAK A., DWORECKI K., JASIK-ŚLĘZAK J., WĄSIK J. 2004. Method to determine the critical concentration Rayleigh number in isothermal passive membrane transport processes. Desalination 168: 397-412. 